\title{
Efficacy of L-carnitine on ribavirin-induced hemolytic anemia in patients with hepatitis $C$ virus infection
}

\author{
Shinya Sato', Kei Moriya', Masanori Furukawa', Soichiro Saikawa', Tadashi Namisaki', Mitsuteru Kitade', \\ Hideto Kawaratani', Kosuke Kaji', Hiroaki Takaya', Naotaka Shimozato', Yasuhiko Sawada', Kenichiro Seki', \\ Koh Kitagawa', Takemi Akahane', Akira Mitoro', Yasushi Okura², Junichi Yamao², and Hitoshi Yoshiji' \\ 'Division of Gastroenterology and Hepatology, Third Department of Internal Medicine, and ${ }^{2}$ Department of Endoscopy, Nara Medical \\ University, Nara, Japan
}

Background/Aims: L-carnitine not only alleviates hyperammonemia and reduces muscle cramps in patients with liver cirrhosis, but also improves anemia in patients with chronic hepatitis and renal dysfunction. This study prospectively evaluated the preventative efficacy of L-carnitine supplementation against hemolytic anemia during antiviral treatment using ribavirin in patients with hepatitis C virus (HCV)-related chronic liver disease.

Methods: A total of 41 patients with chronic hepatitis were consecutively enrolled in this study. Group A $(n=22)$ received sofosbuvir plus ribavirin for 3 months, whereas group $B(n=19)$ was treated with sofosbuvir, ribavirin, and L-carnitine. Hemoglobin concentration changes, the effects of antiviral treatment, and the health status of patients were analyzed using short form-8 questionnaires.

Results: A significantly smaller decrease in hemoglobin concentration was observed in group $B$ compared to group $A$ at every time point. Moreover, the prescribed dose intensity of ribavirin in group $B$ was higher than that of group $A$, resulting in a higher ratio of sustained virological response (SVR) 24 in group B compared with group A. The physical function of patients in group B was also significantly improved compared to group $A$ at the end of antiviral treatment.

Conclusions: L-carnitine supplementation alleviates ribavirin-induced hemolytic anemia in patients with HCV and helps relieve the physical burden of treatment with ribavirin-containing regimens. These advantages significantly increase the likelihood of achieving SVR. (Clin Mol Hepatol 2019;25:65-73)

Keywords: L-carnitine; Hemolytic anemia; Ribavirin; Chronic hepatitis C; Drug therapy

\section{Study Highlights}

In this study, we prospectively evaluated the preventing effect of L-carnitine supplementation during antiviral treatment using ribavirin on a hemolytic anemia in hepatitis C virus related chronic liver disease patients. The decreased level of hemoglobin concentration in the L-carnitine treatment group was significantly smaller than that in the control group through the course of treatment. Additionally, the physical functioning level of patients in the L-carnitine treatment group was significantly better than that in the control group at the end of antiviral treatment. Then, we successfully demonstrated that these effects would increase the opportunity of achieving sustained viral response.

\footnotetext{
Abbreviations:

$\mathrm{CHC}$, chronic hepatitis $\mathrm{C}$; DAAs, direct-acting antivirals; $\mathrm{EOT}$, end of treatment; $H C V$, hepatitis $C$ virus; ITPA, inosine triphosphatase; $L D H$, lactate dehydrogenase; QOL, quality of life; SD, standard deviation; SF-36, short form-36; SF-8, short form-8; SVR, sustained virological response
}

Corresponding author : Kei Moriya

Division of Gastroenterology and Hepatology, Third Department of Internal Medicine, Nara Medical University, 840 Shijo-cho, Kashihara, Nara 634-8521, Japan

Tel: +81-744-22-3051, Fax: +81-744-24-7122

E-mail:moriyak@naramed-u.ac.jp

https://orcid.org/0000-0002-7500-6728 


\section{INTRODUCTION}

In Japan, hepatitis C virus (HCV) infection is the leading cause of liver cirrhosis, its related complications, and the development of hepatocellular carcinoma. ${ }^{1,2}$ The treatment for chronic hepatitis $\mathrm{C}(\mathrm{CHC})$ aimed to eradicate the $\mathrm{HCV}^{3}$ A significantly decreased incidence of disease progression or carcinogenesis indicated a sustained virological response (SVR). ${ }^{3-6} \mathrm{CHC}$ therapy has been dramatically changed with the recent introduction of direct-acting antivirals (DAAs). ${ }^{7-9}$ However, the antiviral treatment for patients with HCV genotype 2 sometimes includes ribavirin. Hemolytic anemia is a commonly observed complication in patients treated with ribavirin-containing regimen; hence, drug discontinuation or dose modifications are recommended.

L-carnitine (4-N-trimethylammonium-3-hydroxybutyric acid), which is involved in $\beta$-oxidation of fatty acids, is a conditionally synthesized nutrient from amino acids methionine and lysine in the brain, liver, and kidney, which is mainly obtained from meat and dairy products. ${ }^{10-12}$ The administration of L-carnitine is an accepted treatment for mitochondrial myopathy and encephalomyopathy, as well as other states of primary and secondary L-carnitine deficiency. ${ }^{13}$ In the field of clinical practice, it has been reported that L-carnitine alleviates hyperammonemia and reduces muscle cramps in patients with liver cirrhosis. ${ }^{14-16}$ Furthermore, Lcarnitine has been recently reported to improve anemia, thrombocytopenia, leukopenia, and immunological dysfunction. ${ }^{17-24}$

This study aimed to evaluate the preventing efficacy of L-carnitine on hemolytic anemia for patients with chronic HCV infection, by which the ribavirin dose is reduced.

\section{PATIENTS AND METHODS}

\section{Patients}

This study has prospectively evaluated the preventing efficacy of L-carnitine supplementation during antiviral treatment using ribavirin on hemolytic anemia in patients with HCV-related chronic liver disease. Chronic HCV infection was confirmed on the basis of the positivity of HCV antibodies and HCV-RNA. The direct sequencing method was used to determine the HCV genotype, as previously reported. ${ }^{25}$

This prospective and randomized clinical study was conducted at the Third Department of Internal Medicine in Nara Medical

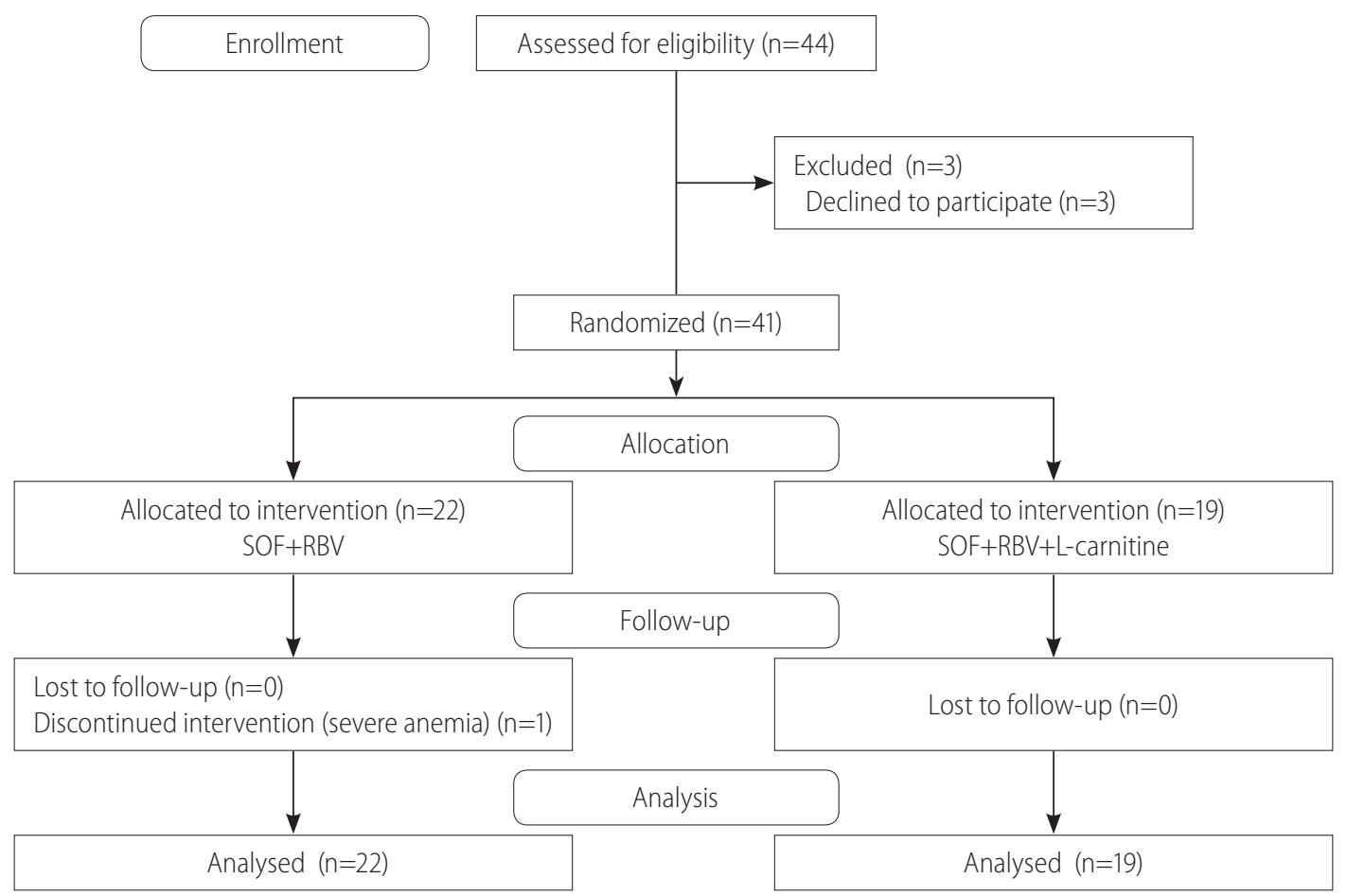

Figure 1. Clinical study profile of additional L-carnitine treatment. This is a prospective, randomized, double-blind clinical trial study. Forty-four patients were enrolled. Three patients who withdrew consent were excluded. Twenty-two patients (group A) received sofosbuvir (SOF)+ribavirin (RBV), whereas 19 patients (group B) were treated with SOF, RBV, and L-carnitine. 
University Hospital between July 2015 and August 2016. The envelope method was used in this study. Each patient chose one of the two envelopes and was enrolled into the group being described. The study was registered at the University Hospital Medical Information Network Clinical Trials Registry (UMIN000026079) on August 15, 2015. A total of 44 patients were primarily registered, and 3 patients who withdrew consent were excluded in this study. A total of 41 patients with chronic hepatitis was consecutively enrolled (Fig. 1). Figure 1 was made according to the CONSORT flow diagram. ${ }^{26}$ In group A, 22 patients received sofosbuvir plus ribavirin for 3 months, whereas in group B, 19 patients were treated with sofosbuvir, ribavirin, and L-carnitine. The baseline clinical characteristics of two groups were described in Table 1. No significant differences in all characteristics between two groups were observed. In group A, 15 patients were treatment naïve, and 3 patients for prior relapse were administered with peginterferon-a2b plus ribavirin combination therapy, whereas 2 of 4 patients for null response were administered with peginterferon$a 2 b$ plus ribavirin combination therapy, and the others received interferon- $a 2 b$ monotherapy. In group B, 16 patients were treatment naïve, and 2 patients for relapse and 1 patient for null response received peginterferon- $a 2 b$ plus ribavirin combination therapy.

Eligible patients who were infected with HCV genotype 2 exhibited a quantifiable serum HCV-RNA level. Cirrhotic patients whose hepatic functional reserves were compatible with the Child-Pugh class A disease were included in this study. Ineligible patients included those who had either another kind of liver disease; any type of cancer; or severe cardiac, pulmonary, or renal diseases. None of the patients exhibited excessive alcohol consumption (male, $>40 \mathrm{~g} /$ day; female, $>20 \mathrm{~g} /$ day). Other causes of exclusion included decompensated cirrhosis, pregnancy, human immunodeficiency virus infection, and contraindications for sofosbuvir and ribavirin therapy.

Table 1. Clinical characteristics of the patients included in this study

\begin{tabular}{|c|c|c|c|}
\hline Clinical characteristics & $\begin{array}{l}\text { Group A }(n=22) \\
\quad(S O F+R B V)\end{array}$ & $\begin{array}{c}\text { Group B ( } n=19) \\
\text { (SOF+RBV+L-carnitine) }\end{array}$ & $P$-value \\
\hline Sex (male/female) & $9 / 13$ & $8 / 11$ & 0.93 \\
\hline Age (year) & $68(64-72.5)$ & $67(56-70)$ & 0.25 \\
\hline White blood cell $\left(\times 10^{2} / \mu \mathrm{L}\right)$ & $46(38-55)$ & $42(37.5-53)$ & 0.66 \\
\hline Hemoglobin (g/dL) & $14.25(13.5-14.5)$ & $13.4(13-14)$ & 0.16 \\
\hline Platelet $\left(\times 10^{4} / \mu \mathrm{L}\right)$ & $13.1(10.6-17.2)$ & $12.6(10.9-15.6)$ & 0.92 \\
\hline Prothrombin time-INR & $1.06(1.03-1.11)$ & $1.07(1.04-1.10)$ & 0.59 \\
\hline Aspartate aminotransferase $(\mathrm{U} / \mathrm{L})$ & $27(22-50)$ & $36(28.5-58.5)$ & 0.33 \\
\hline Alanine aminotransferase (U/L) & $20(16-42)$ & $31(21.5-60)$ & 0.30 \\
\hline Albumin $(\mathrm{g} / \mathrm{dL})$ & $3.9(3.8-4.1)$ & $4.0(3.7-4.1)$ & 0.65 \\
\hline Cholinesterase (U/L) & $281(207-307)$ & $252(222.5-297)$ & 0.42 \\
\hline Total-bilirubin (mg/dL) & $0.8(0.62-0.97)$ & $0.7(0.55-0.8)$ & 0.64 \\
\hline HCV-RNA (log IU/mL) & $6.2(5.3-6.7)$ & $5.1(4.55-6.2)$ & 0.06 \\
\hline Genotype 2A/2B & $16 / 6$ & $15 / 4$ & 0.64 \\
\hline $\mathrm{P}-\mathrm{III-P}(\mathrm{U} / \mathrm{mL})$ & $0.9(0.9-1.0)$ & $1.15(0.8-1.35)$ & 0.19 \\
\hline Type IV collagen (ng/mL) & $4.1(3.3-6.4)$ & $4.4(4-6.7)$ & 0.39 \\
\hline a-fetoprotein (ng/mL) & $2.6(1.85-3.35)$ & $3(2.5-5.25)$ & 0.54 \\
\hline Liver biopsy (yes/no) & $18 / 22$ & 15/19 & 0.81 \\
\hline $\mathrm{A} 1 / \mathrm{A} 2 / \mathrm{A} 3$ & 0/8/0 & 0/6/0 & 0.79 \\
\hline F1/F2/F3/F4 & $0 / 3 / 1 / 2$ & $0 / 3 / 1 / 1$ & 0.97 \\
\hline \multicolumn{4}{|l|}{ Previous treatment } \\
\hline Naïve/Relapsed/Null responded & $15 / 3 / 4$ & $16 / 2 / 1$ & 0.40 \\
\hline
\end{tabular}

Numerical variables were indicated as the median with interquartile range.

SOF, sofosbuvir; RBV, ribavirin; INR, International Normalized Ratio; HCV, hepatitis C virus; P-III-P, type III procollagen-N-peptide; A, inflammation; F, fibrosis. 


\section{Treatments}

Patients in group $A(n=22)$ received sofosbuvir plus ribavirin for 3 months, whereas those in group $B(n=19)$ were treated with sofosbuvir, ribavirin, and L-carnitine for the same period. The daily dose of sofosbuvir and L-carnitine was fixed as 400 and 1,500 mg, respectively, and that of ribavirin was determined by the body weight of each patient: (i) $600 \mathrm{mg}$ for a body weight of less than $60 \mathrm{~kg}$, (ii) $800 \mathrm{mg}$ for between 60 and $80 \mathrm{~kg}$, and (iii) 1,000 mg for over $80 \mathrm{~kg}$. The dose of ribavirin and its duration were determined according to the clinical guidelines of the Japan Society of Hepatology. ${ }^{27}$ An SVR was achieved in patients with undetectable levels of HCV-RNA at 24 weeks after the end of treatment (EOT). Patients were carefully observed from the start of treatment to 6 months after medication (SVR24). Their health statuses were quantified physically and mentally using the short form-8 (SF-8) questionnaires before medication, at the EOT, and at the SVR24 time point. ${ }^{28}$ The short form-36 (SF-36) Health Survey, developed in the Medical Outcomes Study, is the most popular instrument to measure health-related quality of life (QOL). However, the SF-36 is quite lengthy, which limits its use. Therefore, the SF-8, a shortened version of SF-36 and the shortest SF Health Survey, is preferred by a number of researchers. ${ }^{29}$

\section{Statistical analysis}

This study has planned a continuous response variable from independent control and experimental subjects with one control per experimental subject. In the previous study, the response within each subject group was normally distributed with a standard deviation (SD) of 3.3. If the true difference in the experimental and control means is 3, 20 experimental subjects and 20 control subjects need to be examined to be able to reject the null hypothesis that the population means of the experimental and control groups are equal with a probability (power) of 0.8 . Type I error probability associated with the test of this null hypothesis is 0.05 . The numerical variables were expressed as the mean $\pm S D$. Quantitative parameters were compared using the Mann-Whitney $U$ test. Categorical parameters were compared using Pearson's chi-square test or Fisher's exact test, as applicable. And then for each outcome of lactate dehydrogenase (LDH) and hemoglobin, repeatedmeasures univariate analysis of variance was conducted to assess the effects. A $P$-value less than 0.05 was considered statistically significant.

This study was approved by the Nara Medical University Ethics
Committee and was conducted according to the Declaration of Helsinki. The present study protocol was registered as a clinical trial (UMIN000026079, https://upload.umin.ac.jp/), and written informed consent was obtained from all patients.

\section{RESULTS}

\section{Patient characteristics}

Patient characteristics are shown in Table 1. The baseline characteristics of the two groups, including gender, age, leukocyte counts, hemoglobin concentrations, platelet counts, prothrombin time, serum aspartate aminotransferase level, alanine aminotransferase level, albumin, cholinesterase, and total bilirubin, were comparable, with no significant differences between the groups. In addition, no significant differences were observed between HCV-RNA, genotype ratio, and previous histories of antiviral treatments. Three cirrhotic patients were included in this study, two of which were treated in group $A$ and the other was in group B.

\section{The effect of L-carnitine supplementation on hemolytic anemia}

Changes of hemoglobin concentration in both groups are shown in Fig. 2. Hemoglobin concentrations in both groups were actually decreased compared with each baseline level at 4 weeks after the start of antiviral treatment. However, the decreased level of hemoglobin concentration was significantly lower in group $B$ than in group A at every time point, which suggested that L-carnitine supplementation helps alleviate ribavirin-induced hemolytic anemia effectively. Consistently, the serum LDH level of patients in group $A$ was significantly higher than that in group $B$ at both 8 and 12 weeks after the start of treatment (Fig. 3). No significant changes were observed in the levels of other biochemical parameters through the course of antiviral treatment in each group (data not shown). In group B, no severe adverse events were observed to develop; however, two cases of abdominal distensions occurred, which are considered as mild adverse events.

\section{The effect of antiviral treatment}

In group A, 17 patients completed the protocol without dose modification of ribavirin, whereas 4 patients were recommended to modify their ribavirin dose, due to the occurrence of mild he- 
molytic anemia, and a patient was dropped out of the treatment because of severe anemia at the sixth week. In group B, all patients accomplished the antiviral protocol with full dose of ribavirin. In summary, the dose intensity of ribavirin in group $B$ was higher than that of group $A(100 \%$ vs. $91.2 \%)$.

Two patients in group A could not achieve SVR24, one of which was dropped out of the treatment because of severe anemia, and the other was inevitable to ribavirin dose modification. Both of them did not develop liver cirrhosis. On the other hand, all patients in group B achieved SVR24. As a result, the ratio of SVR24 in group B was higher than that in group A (100\% vs. $90.9 \%$ ).

\section{Patient's QOL}

The health status of participants was also assessed according to the physical and mental aspects using the SF-8 questionnaires, excluding a patient who had been dropped out from this study. No significant differences were observed between groups at three different time points: before medication, EOT, and SVR24. However, there was a marginal difference in the physical component summary of patients at the EOT (Fig. 4A), whereas no significant differences were observed between groups in mental component summary at three time points (Fig. 4B). Then, a more detailed assessment was performed on each scale of physical components in both groups at the EOT, and it was found that physical functioning of patients in group $B$ was significantly improved than that in group A (Fig. 4C). This result seemed to indicate the physical burden of patients treated with the ribavirin-containing regimen.

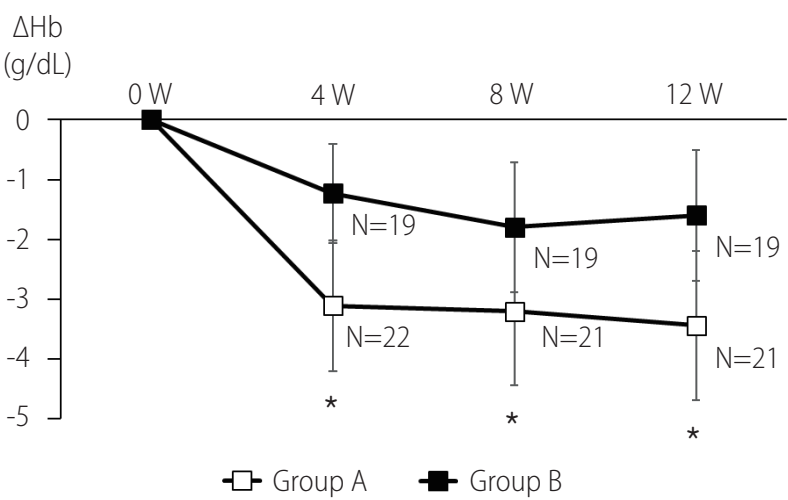

Figure 2. Decrese in hemoglobin ( $\mathrm{Hb}$ ) concentration. A significantly smaller decrement in hemoglobin concentration was observed in group B compared to group A throughout the observation period. W, week(s). ${ }^{*} P<0.05$.

\section{DISCUSSION}

$\mathrm{HCV}$ infection is the most frequent cause of chronic liver disease in Japan, and hemolytic anemia may be a major complication in $\mathrm{HCV}$-infected patients treated with ribavirin-containing regimen. ${ }^{30}$ Drug discontinuation or dose modifications are recommended for patients who developed hemolytic anemia, to facilitate an effective eradication of the $\mathrm{HCV}^{31,32} \mathrm{~L}$-carnitine has been recently reported to improve the hematological pattern of patients with HCV infection and renal failure. ${ }^{17-24}$ However, its preventing efficacy for patients treated with the combination of DAAs and ribavirin remains largely unclear. This study evaluated the preventing efficacy of L-carnitine for patients with chronic HCV infection who developed hemolytic anemia by which the ribavirin dose is reduced.

Hemolytic anemia is likely related to extensive ribavirin accumulation in erythrocytes, possibly following active unidirectional transmembrane transport. Ribavirin exerts its toxicity through the inhibition of intracellular energy metabolism and oxidative membrane damage, leading to an accelerated extravascular hemolysis by the reticuloendothelial system, ${ }^{33}$ though the ribavirin is known as to be an important factor for effective antiviral therapy. ${ }^{31}$

By the way, in thalassemia patients, anemia was induced by an impairment of the stabilization for membrane in erythrocytes and L-carnitine were known to modulate the apoptotic process. ${ }^{34}$ On the other hand, in patients with chronic kidney disease, anemia progression occurred through the mechanism of the excessive degradation of serum erythropoietin, and L-carnitine increased the efficacy of erythropoietin. ${ }^{19}$ In such a situation, the protective effect of L-carnitine on erythrocytes from oxidative stress and the stabilization to their membranes where latent peroxidative dam-

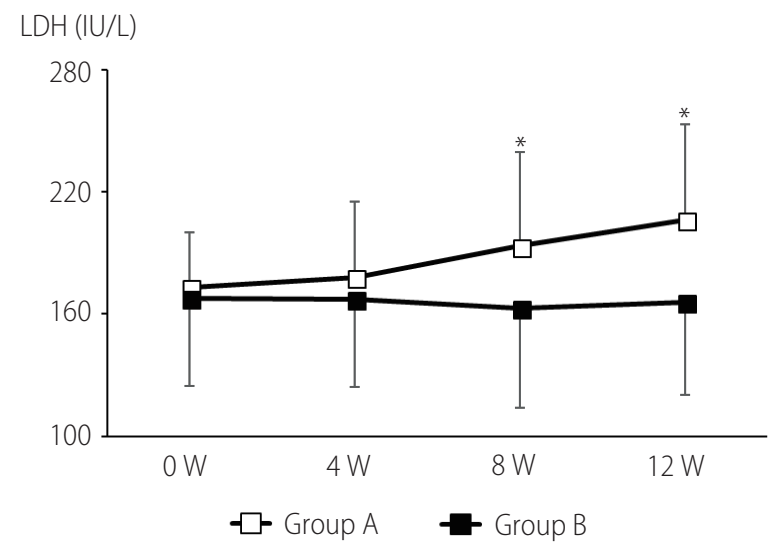

Figure 3. Changes in serum lactate dehydrogenase (LDH). Group A serum $L D H$ was increased throughout antiviral treatment, whereas that in group $B$ was unchanged. $W$, week(s). ${ }^{*} P<0.05$. 

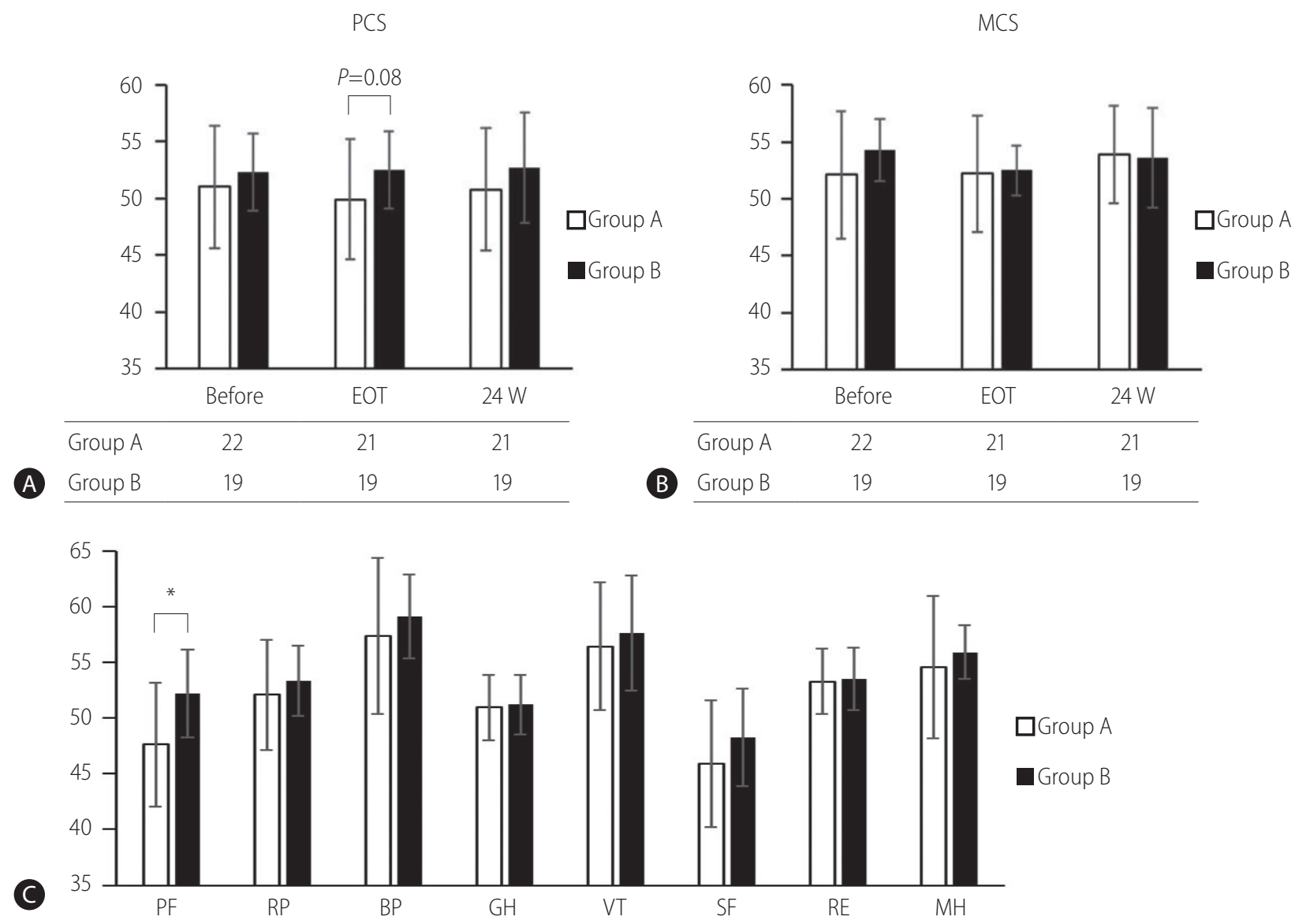

Figure 4. Patient quality of life on the SF-8 Health Survey questionnaire. (A, B) At the end of treatment (EOT), a marginal difference was observed in the physical component summary of patients, whereas no significant differences were observed between groups in the mental component summary at three different time points: before medication, at the EOT, and sustained virological response 24. (C) Each scale was assessed in both groups at the EOT. Physical function was significantly improved for patients in group B compared with group A. PCS, physical component summary; W, weeks; MCS, mental component summary; PF, physical functioning; RP, role physical; BP, bodily pain; GH, general health perception; VT, vitality; SF, social functioning; $\mathrm{RE}$, role emotional; $\mathrm{MH}$, mental health. ${ }^{*} P<0.05$.

age has occurred, was previously reported. ${ }^{35-37}$ The detailed mechanism of the L-carnitine efficacy on the ribavirin-induced hemolytic anemia in the patients with chronic HCV infection should be elucidated in the near future, but the most probable scenario would be that L-carnitine effectively makes the membrane in erythrocytes equable and alleviates the oxidative stress in them.

On the basis of the results of this study, L-carnitine supplementation effectively alleviates ribavirin-induced hemolytic anemia, and patients treated with L-carnitine successfully kept their ribavirin adherence and consequently achieved SVR24. This is compatible with the molecular mechanism in which L-carnitine effectively makes the membrane in erythrocytes equable and alleviates the oxidative stress.

Moreover, L-carnitine supplementation may relieve the physical burden of patients during treatment with ribavirin-containing regimen. According to Tomassini et al., acetyl-L-carnitine contributed to the improvement of energetic metabolism in myocardial and musculoskeletal tissue, which led to reduction of the severity of physical fatigue in sample subjects. ${ }^{38}$

Several limitations were also identified in this study. First, this study failed to evaluate the genetic polymorphism of inosine triphosphatase (ITPA), which was reported to be associated with the progression of hemolytic anemia, ${ }^{39}$ due to the lack of consensus on ethical issues. ITPA is a determinant used in genetic testing, and an agreement from each participant of this study was hardly obtained. Second, serum carnitine and ribavirin levels of patients in both groups were not monitored because of serum sample shortage. In the previous report, total carnitine levels 
were significantly lower in patients with CHC than in healthy controls when adjusted for fat mass. ${ }^{40}$ Moreover, the present study utilized an open-label design and included a relatively small sample size. Future studies should include a large number of patients to confirm results in this study. In addition, only the Japanese population was involved in this study; therefore, additional investigations on different ethnic populations are desired to determine the preventing efficacy of L-carnitine on hemolytic anemia for patients with chronic HCV infection.

However, to the best of our knowledge, this is the first prospective and randomized clinical trial assessing the effect of L-carnitine supplementation on ribavirin-induced hemolytic anemia for patients with HCV infection. Globally, some existing DAA regimens using ribavirin has been mainly selected for patients with decompensated cirrhosis and those who relapsed after prior DAA monotherapy. ${ }^{41,42}$ In recent years, a newly established regimen, sofosbuvir-velpatasvir with ribavirin, is going to be approved for patients with decompensated cirrhosis in Japan, and L-carnitine would be useful in these cases as a supportive therapy.

In summary, L-carnitine supplementation significantly alleviates ribavirin-induced hemolytic anemia in patients with HCV and may relieve the physical burden of patients during the treatment of the ribavirin-containing regimen. These advantages would increase the chance of achieving SVR and hopefully decrease the risk of hepatocellular carcinoma.

\section{Availability of data and materials}

The datasets used and/or analyzed during the present study are available from the corresponding author on reasonable request.

\section{Authors' contribution}

Concept and design: Shinya Sato, Kei Moriya, Takemi Akahane, and Hitoshi Yoshiji.

Clinical data collection: Soichiro Saikawa, Kosuke Kaji, Hiroaki Takaya, Naotaka Shimozato, Yasuhiko Sawada, Kenichiro Seki, Koh Kitagawa, Akira Mitoro, Yasushi Okura, and Junichi Yamao.

Writing of article: Shinya Sato.

Editing of article: Kei Moriya.

Data analysis: Masanori Furukawa, Tadashi Namisaki, Mitsuteru Kitade, and Hideto Kawaratani.

All the authors have seen and approved.

\section{Acknowledgments}

The authors would like to thank patients who participated to this study.

\section{Financial support}

The study was supported in part by a grant-in-aid for research from Nara Medical University.

\section{Conflicts of Interest}

Authors declare no conflicts of interest.

\section{REFERENCES}

1. Takeda H, Takai A, Inuzuka T, Marusawa H. Genetic basis of hepatitis virus-associated hepatocellular carcinoma: linkage between infection, inflammation, and tumorigenesis. J Gastroenterol 2017:52:26-38.

2. Martel-Laferrière V, Bichoupan K, Dieterich DT. Hepatitis C directacting antiviral agents: changing the paradigm of hepatitis $C$ treatment in HIV-infected patients. J Clin Gastroenterol 2014;48:106112.

3. Wang LS, D'Souza LS, Jacobson IM. Hepatitis C-A clinical review. J Med Virol 2016;88:1844-1855.

4. van der Meer AJ, Berenguer M. Reversion of disease manifestations after HCV eradication. J Hepatol 2016;65(1 Suppl):S95-S108.

5. Gutierrez JA, Lawitz EJ, Poordad F. Interferon-free, direct-acting antiviral therapy for chronic hepatitis C. J Viral Hepat 2015;22:861870.

6. Jacobson IM. The HCV treatment revolution continues: resistance considerations, pangenotypic efficacy, and advances in challenging populations. Gastroenterol Hepatol (N Y) 2016;12(10 Suppl 4):1-11.

7. Asselah T, Marcellin P. Interferon free therapy with direct acting antivirals for HCV. Liver Int 2013;33 Suppl 1:93-104.

8. Fried MW, Buti M, Dore GJ, Flisiak R, Ferenci P, Jacobson I, et al. Once-daily simeprevir (TMC435) with pegylated interferon and ribavirin in treatment-naïve genotype 1 hepatitis C: the randomized PILLAR study. Hepatology 2013;58:1918-1929.

9. Gane EJ, Stedman CA, Hyland RH, Ding X, Svarovskaia E, Symonds WT, et al. Nucleotide polymerase inhibitor sofosbuvir plus ribavirin for hepatitis C. N Engl J Med 2013;368:34-44.

10. Kendler BS. Carnitine: an overview of its role in preventive medicine. Prev Med 1986;15:373-390.

11. Malaguarnera M. Carnitine derivatives: clinical usefulness. Curr Opin Gastroenterol 2012;28:166-176.

12. Thangasamy $T$, Subathra M, Sittadjody S, Jeyakumar P, Joyee AG, Mendoza $E$, et al. Role of L-carnitine in the modulation of immune response in aged rats. Clin Chim Acta 2008;389:19-24.

13. Campos Y, Huertas R, Bautista J, Gutiérrez E, Aparicio M, Lorenzo $G$, et al. Muscle carnitine deficiency and lipid storage myopathy in patients with mitochondrial myopathy. Muscle Nerve 1993;16:778- 
781.

14. Nakanishi H, Kurosaki M, Tsuchiya K, Nakakuki N, Takada H, Matsuda $\mathrm{S}$, et al. L-carnitine reduces muscle cramps in patients with cirrhosis. Clin Gastroenterol Hepatol 2015;13:1540-1543.

15. Shiraki M, Shimizu M, Moriwaki H, Okita K, Koike K. Carnitine dynamics and their effects on hyperammonemia in cirrhotic Japanese patients. Hepatol Res 2017;47:321-327.

16. Cecere A, Ciaramella F, Tancredi L, Romano C, Gattoni A. Efficacy of $\mathrm{L}$-carnitine in reducing hyperammonaemia and improving neuropsychological test performance in patients with hepatic cirrhosis: results of a randomised trial. Clin Drug Investig 2002;22(Suppl 1):714.

17. Hurot JM, Cucherat M, Haugh M, Fouque D. Effects of L-carnitine supplementation in maintenance hemodialysis patients: a systematic review. J Am Soc Nephrol 2002;13:708-714.

18. Weinhandl ED, Rao M, Gilbertson DT, Collins AJ, Pereira BJ. Protective effect of intravenous levocarnitine on subsequent-month hospitalization among prevalent hemodialysis patients, 1998 to 2003. Am J Kidney Dis 2007;50:803-812.

19. Matsumoto Y, Amano I, Hirose S, Tsuruta Y, Hara S, Murata M, et al. Effects of $L$-carnitine supplementation on renal anemia in poor responders to erythropoietin. Blood Purif 2001;19:24-32.

20. Sotirakopoulos N, Athanasiou G, Tsitsios T, Mavromatidis K. The influence of I-carnitine supplementation on hematocrit and hemoglobin levels in patients with end stage renal failure on CAPD. Ren Fail 2002;24:505-510.

21. Abdallah Y, Gligorievski D, Kasseckert SA, Dieterich L, Schäfer M, Kuhlmann CR, et al. The role of poly(ADP-ribose) polymerase (PARP) in the autonomous proliferative response of endothelial cells to hypoxia. Cardiovasc Res 2007;73:568-574.

22. Izgüt-Uysal VN, Agaç A, Karadogan I, Derin N. Peritoneal macrophages function modulation by L-carnitine in aging rats. Aging Clin Exp Res 2004;16:337-341.

23. Saluk-Juszczak J, Olas B, Wachowicz B, Glowacki R, Bald E. Lcarnitine modulates blood platelet oxidative stress. Cell Biol Toxicol 2010;26:355-365.

24. Malaguarnera G, Pennisi M, Gagliano C, Vacante M, Malaguarnera $M$, Salomone $S$, et al. Acetyl-L-carnitine supplementation during HCV therapy with pegylated interferon-a $2 b$ plus ribavirin: effect on work performance; a randomized clinical trial. Hepat Mon 2014;14:e11608.

25. Tamura K, Stecher G, Peterson D, Filipski A, Kumar S. MEGA6: molecular evolutionary genetics analysis version 6.0. Mol Biol Evol 2013;30:2725-2729.

26. Consolidated Standards of Reporting Trials (CONSORT). The CONSORT flow diagram. CONSORT web site, <http://www.consortstatement.org/consort-statement/flow-diagram>. Accessed 15 Aug 2018
27. Editors of the Drafting Committee for Hepatitis Management Guidelines: The Japan Society of Hepatology. Guidelines for the management of hepatitis C virus infection: first edition, May 2012, the Japan Society of Hepatology. Hepatol Res 2013;43:1-34.

28. Ware JE Jr, Bjorner JB, Kosinski M. Practical implications of item response theory and computerized adaptive testing: a brief summary of ongoing studies of widely used headache impact scales. Med Care 2000;38(9 Suppl):॥173-II82.

29. Tokuda Y, Okubo T, Ohde S, Jacobs J, Takahashi O, Omata F, et al. Assessing items on the SF-8 Japanese version for health-related quality of life: a psychometric analysis based on the nominal categories model of item response theory. Value Health 2009;12:568-573.

30. De Franceschi L, Fattovich G, Turrini F, Ayi K, Brugnara C, Manzato F, et al. Hemolytic anemia induced by ribavirin therapy in patients with chronic hepatitis C virus infection: role of membrane oxidative damage. Hepatology 2000;31:997-1004.

31. Hadziyannis SJ, Sette H Jr, Morgan TR, Balan V, Diago M, Marcellin $\mathrm{P}$, et al. Peginterferon-alpha2a and ribavirin combination therapy in chronic hepatitis C: a randomized study of treatment duration and ribavirin dose. Ann Intern Med 2004;140:346-355.

32. Luca C, Grigore L, Vâță A, Dorobăț C. Adverse reactions of different treatments in chronic hepatitis C. Rev Med Chir Soc Med Nat lasi 2009;113:991-995.

33. Russmann S, Grattagliano I, Portincasa P, Palmieri VO, Palasciano G. Ribavirin-induced anemia: mechanisms, risk factors and related targets for future research. Curr Med Chem 2006;13:3351-3357.

34. El-Beshlawy A, Seoud H, Ibrahim A, Youssry I, Gabre H, Isma'eel H, et al. Apoptosis in thalassemia major reduced by a butyrate derivative. Acta Haematol 2005;114:155-159.

35. Yeşilipek MA, Yeğin 0. Interferon-alpha therapy for refractory idiopathic thrombocytopenic purpura in children. Turk J Pediatr 1997:39:173-176.

36. Palmieri L, Ronca F, Malengo S, Bertelli A. Protection of beta-thalassaemic erythrocytes from oxidative stress by propionyl carnitine. Int J Tissue React 1994;16:121-129.

37. Tsukuda Y, Suda G, Tsunematsu S, Ito J, Sato F, Terashita K, et al. Anti-adipogenic and antiviral effects of I-carnitine on hepatitis $C$ virus infection. J Med Virol 2017;89:857-866.

38. Tomassini V, Pozzilli C, Onesti E, Pasqualetti P, Marinelli F, Pisani $A$, et al. Comparison of the effects of acetyl L-carnitine and amantadine for the treatment of fatigue in multiple sclerosis: results of a pilot, randomised, double-blind, crossover trial. J Neurol Sci 2004:218:103-108.

39. Maan R, van der Meer AJ, Brouwer WP, Plompen EP, Sonneveld MJ, Roomer $R$, et al. ITPA polymorphisms are associated with hematological side effects during antiviral therapy for chronic HCV infection. PLoS One 2015;10:e0139317.

40. Anty R, Marjoux S, Bekri S, DeGalleani L, Dainese R, Gelsi E, et al. 
Shinya Sato, et al.

Plasma carnitine is associated with fatigue in chronic hepatitis C but not in the irritable bowel syndrome. Aliment Pharmacol Ther 2011;33:961-968.

41. Curry MP, O'Leary JG, Bzowej N, Muir AJ, Korenblat KM, Fenkel JM, et al. Sofosbuvir and velpatasvir for HCV in patients with decom- pensated cirrhosis. N Engl J Med 2015;373:2618-2628.

42. Gane EJ, Shiffman ML, Etzkorn K, Morelli G, Stedman CAM, Davis $M N$, et al. Sofosbuvir-velpatasvir with ribavirin for 24 weeks in hepatitis $C$ virus patients previously treated with a direct-acting antiviral regimen. Hepatology 2017;66:1083-1089. 